\title{
Increased accuracy of ligand sensing by receptor diffusion on cell surface
}

\author{
Gerardo Aquino and Robert G. Endres \\ Division of Molecular Biosciences and Centre for Integrated Systems Biology at Imperial College, \\ Imperial College London, SW7 2AZ, London, UK
}

(Dated: September 30, 2018)

\begin{abstract}
The physical limit with which a cell senses external ligand concentration corresponds to the perfect absorber, where all ligand particles are absorbed and overcounting of same ligand particles does not occur. Here we analyze how the lateral diffusion of receptors on the cell membrane affects the accuracy of sensing ligand concentration. Specifically, we connect our modeling to neurotransmission in neural synapses where the diffusion of glutamate receptors is already known to refresh synaptic connections. We find that receptor diffusion indeed increases the accuracy of sensing for both the glutamate AMPA and NDMA receptors, although the NMDA receptor is overall much noiser. We propose that the difference in accuracy of sensing of the two receptors can be linked to their different roles in neurotransmission. Specifically, the high accuracy in sensing glutamate is essential for the AMPA receptor to start membrane depolarization, while the NMDA receptor is believed to work in a second stage as a coincidence detector, involved in long-term potentiation and memory.
\end{abstract}

PACS numbers:

\section{INTRODUCTION}

Biological cells live in very noisy environments from which they receive many different stimuli. The survival of a cell highly depends on its ability to respond to such stimuli and to adapt to changes in the environment. A fundamental role in this task is played by membrane receptors, proteins on the cell surface which are able to bind external ligand molecules and trigger signal transduction pathways. Although the precision with which a cell can measure the concentration of a specific ligand is negatively affected by many sources of noise 1-4], several examples exist in which such measurements are performed with surprisingly high accuracy. In bacterial chemotaxis for instance, the bacterium Escherichia coli can respond to changes in concentration as low as $3.2 \mathrm{nM}$ [5], corresponding to only three molecules in the volume of a cell. High accuracy is observed also in spatial sensing by single cell eukaryotic organisms as e.g. in the slime mold Dictyostelium discoideum, which is able to sense a concentration difference of only $1-5 \%$ across the cell diameter [6], and in Saccharomyces cerevisiae (budding yeast), which is able to orient growth in a gradient of $\alpha$ pheromone mating factor down to estimated $1 \%$ receptor occupancy difference across the cell [7]. Spatial sensing is also efficiently performed by growing neurons, lymphocytes, neutrophils and other cells of the immune system.

There has been substantial progress in our theoretical understanding of the accuracy of concentration sensing. In 1977, Berg and Purcell were the first to point out that ligand sensing is limited by ligand diffusion, producing a "counting noise" at the receptors [8]. Bialek and Setayeshgar 9] (and later others 10]) used the Fluctuation Dissipation Theorem (FDT) to separate the contributions from random binding/unbinding events from rebinding due to diffusion of ligand molecules. Subsequently, Wingreen and Endres showed that the perfect

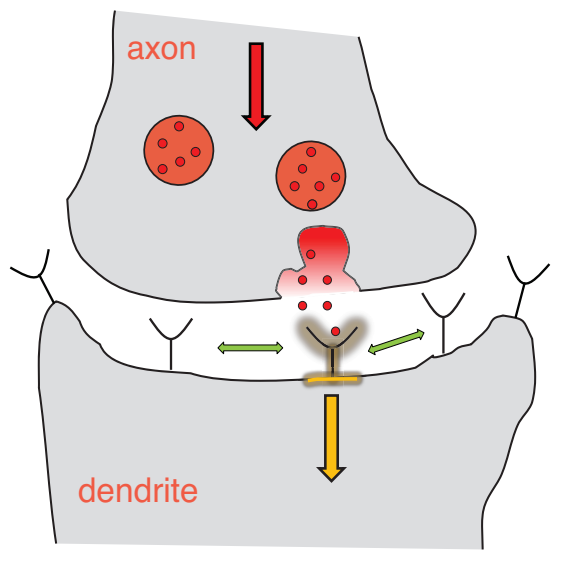

FIG. 1: (Color online) Synaptic junction during neurotransmission. A vesicle containing a neurotransmitter (e.g. glutamate for excitatory synapses), releases its content into the synaptic cleft. Receptors on the post-synaptic surface bind the neurotransmitter, increasing the likelihood of the propagation of the electric impulse (action potential). Red arrow indicates the incoming potential, yellow arrow the set of reactions leading to propagation of potential, small green arrows indicate receptor mobility.

absorber is the absolute fundamental limit of ligand sensing accuracy; it does not suffer from ligand unbinding and rebinding noise [10, 11].

In previous work we analyzed the contribution of endocytosis, i.e. the internalization of cell-surface receptors, to the accuracy of external ligand concentration sensing [12]. We showed with a simple model that internalization, by making the cell act as an absorber of ligand, increases sensing accuracy by reducing the noise from rebinding of already measured ligand molecules. In this paper we 
consider the effect of lateral diffusion of receptors on the accuracy of ligand concentration sensing.

In a situation in which ligand sensing is concentrated in a specific region on the cell membrane (signaling hotspot), receptors bind ligand molecules locally but may release them remotely from the region of interest, thus preventing those particles from rebinding locally. Noise from overcounting could therefore be reduced, potentially increasing the accuracy of sensing.

A potential implementation of such localized sensing occurs in neural synapses, responsible for transmission of action potentials and short-term synaptic plasticity (see Fig. 1 for a schematic description). At such critical places in the central nervous system, the accuracy of sensing ought to be important [13]. At a synapse the propagation of an action potential arriving from the presynaptic neuron causes the opening of ion channels on the pre-synaptic membrane which increases calcium-ion inflow [14]. Calcium ions trigger a biochemical cascade which results in the formation of vesicles containing neurotransmitter, e.g. glutamate, in the case of excitatory synapses. One or more vesicles eventually fuse with the membrane at a point on the pre-synaptic surface to form a fusion pore. Glutamate is subsequently released and diffuses into the synaptic cleft, reaching the receptors on the post-synaptic surface on the opposite side of the cleft. These receptors, mainly the AMPA and NMDA receptors (named after the agonists $\alpha$-amino-3-hydroxy-5-methyl4-isoxazolepropionic acid and N-methyl-D-aspartic acid respectively), cause ion channels on the post-synaptic membrane to open, facilitating the propagation of the action potential.

The AMPA receptor (AMPAR) has a tetrameric structure with four binding sites for glutamate. Binding glutamate on two sites leads to conformational change and opening of a pore. More occupied binding sites imply a higher current through the channel, mainly of calcium, sodium and potassium ions. The NMDA receptor (NMDAR) has a tetrameric structure as well, but its channel needs coincidental binding of glutamate and glycine molecules on dedicated sites for it to open. A small membrane depolarization is furthermore necessary to clear out the magnesium ions blocking the channel. This third condition makes the NMDA receptor a type of coincidence detector for membrane depolarization and synaptic transmission, playing an important role in memory and learning. Interestingly, AMPA receptors diffuse on the post-synaptic surface unusually fast, especially when ligand is bound [15]. In addition to refreshing the synaptic plasticity [16], such diffusion may also increase the accuracy of sensing in neurotransmission.

This paper is organized as follows: in Sec. II we review the case of a single immobile receptor; in Sec. III we consider receptor diffusion on the membrane and derive the dynamical equations determining the spatial concentration of ligand and the occupancy of the receptor. In Sec. IV we derive the stationary solution for the receptor occupancy, and in Sec. V, using a non-equilibrium ap- proach based on the effective temperature, we derive the accuracy of sensing. In Section VI we describe how our theoretical results connect to neurotransmission in neural synapses. The final section is devoted to an overall discussion including cases of further biological relevance. Technical details are provided in two appendices.

\section{REVIEW OF THE SINGLE RECEPTOR}

In this section we review previous results for a single, immobile receptor. As depicted in Fig. 2, such a receptor can bind and release ligand with rates $k_{+} \bar{c}$ and $k_{-}$, respectively. The kinetics for the occupancy $n(t)$ of the receptor are therefore given by

$$
\frac{d n(t)}{d t}=k_{+} \bar{c}[1-n(t)]-k_{-} n(t),
$$

where the concentration of ligand, $\bar{c}$, is assumed uniform and constant. The steady-state solution for the receptor occupancy is given by

$$
\bar{n}=\frac{\bar{c}}{\bar{c}+K_{D}}
$$

with $K_{D}=k_{-} / k_{+}$the ligand dissociation constant. The rates of binding and unbinding are related to the (negative) free energy $F$ of binding through the detailed balance

$$
\frac{k_{+} \bar{c}}{k_{-}}=e^{\frac{F}{T}}
$$

with $T$ the temperature in energy units. In the limit of very fast ligand diffusion, i.e. when a ligand molecule is immediately removed from the receptor after unbinding, the receptor dynamics are effectively decoupled from the diffusion of ligand molecules and hence, diffusion does not need to be included explicitly.

Following Bialek and Setayeshgar [9], the accuracy of sensing is obtained by applying the FDT [17], which relates the spectrum of fluctuations in occupancy to the linear response of the receptor occupancy to a perturbation in the receptor binding energy. Furthermore, at equilibrium the fluctuations in occupancy can be directly related to the uncertainty in ligand concentration using Eq. (2). After time-averaging over a duration $\tau$ much larger than the correlation time of the binding and unbinding events, the normalized uncertainty of sensing is given by $[9,18$ ]

$$
\frac{\left\langle(\delta c)^{2}\right\rangle_{\tau}}{\bar{c}^{2}}=\frac{2}{k_{+} \bar{c}(1-\bar{n}) \tau} \rightarrow \frac{1}{2 \pi D_{3} \bar{c} s \tau}
$$

where the right-hand side is obtained for diffusion-limited binding [18], i.e. when $k_{+} \bar{c}(1-\bar{n}) \rightarrow 4 \pi \bar{c} D_{3} s$, with $D_{3}$ the diffusion constant and $s$ the dimension of the (spherical) receptor. Eq. (4) shows that the uncertainty is limited by the random binding and unbinding of ligand. 


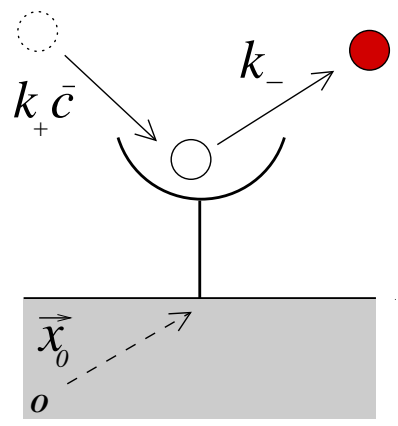

FIG. 2: (Color online) Single receptor, immobile at position $\vec{x}_{0}$, binds and unbinds ligand with rates $k_{+} \bar{c}$ and $k_{-}$, respectively.

The accuracy of sensing is defined as the inverse of the uncertainty.

In the case where diffusion of ligand is slow, ligand binding to the receptor is perturbed [9]. The kinetics of receptor occupancy and ligand concentration are described by

$$
\begin{aligned}
& \frac{d n(t)}{d t}=k_{+} c\left(\vec{x}_{0}, t\right)[1-n(t)]-k_{-} n(t) \\
& \frac{\partial c(\vec{x}, t)}{\partial t}=D_{3} \nabla^{2} c(\vec{x}, t)-\delta\left(\vec{x}-\vec{x}_{0}\right) \frac{d n(t)}{d t},
\end{aligned}
$$

where $\vec{x}_{0}$ indicates the position of the receptor. In the last term in the second equation, the Dirac delta function $\delta\left(x-\vec{x}_{0}\right)$ describes a sink or source of ligand at $\vec{x}_{0}$ corresponding to ligand binding or unbinding from the receptor, respectively.

Following a similar procedure as in the previous case, the uncertainty of sensing is given by [9, 18]

$$
\begin{aligned}
\frac{\left\langle(\delta c)^{2}\right\rangle_{\tau}}{\bar{c}^{2}} & =\frac{2}{k_{+} \bar{c}(1-\bar{n}) \tau}+\frac{1}{\pi D_{3} \bar{c} s \tau} \\
& \rightarrow \frac{3}{2 \pi D_{3} \bar{c} s \tau},
\end{aligned}
$$

where the first term on the right-hand side of Eq. (6a) is the same as in Eq. (44), while the second term is the increase in uncertainty due to diffusion. This term accounts for the additional measurement uncertainty from rebinding of previously bound ligand to the receptor. For diffusion-limited binding, one obtains Eq. (6b) [18].

Comparison of Eqs. (44) and (6) shows that removal of previously bound ligand by fast diffusion increases the accuracy of sensing, since the same ligand molecule is never measured more than once.

The uncertainty in Eq. (44) can be further reduced by a factor of two, consequently increasing the accuracy of sensing by the same factor, by considering the fundamental limit. In this limit, ligand particles are absorbed, and hence only binding noise contributes. For diffusion-limited binding, the fundamental limit is given by $[8,10,11]$

$$
\frac{\left\langle(\delta c)^{2}\right\rangle_{\tau}}{\bar{c}^{2}}=\frac{1}{4 \pi D_{3} \bar{c} s \tau}
$$

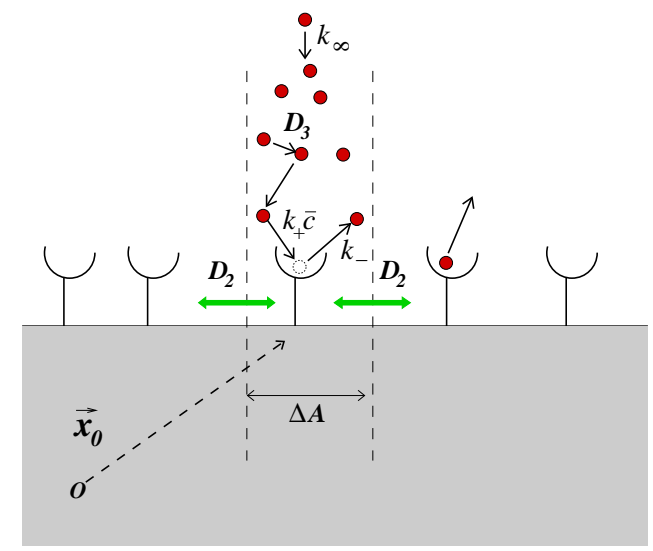

FIG. 3: (Color online) Ligand-receptor binding by mobile receptors characterized by diffusion coefficient $D_{2}$ (green arrows). Ligand (red dots) is concentrated only in a small area $\Delta A$ around $\vec{x}_{0}$ due to e.g. local release of glutamate from vesicle fusion pore with rate $k_{\infty}$. Ligand can bind to receptors at $\vec{x}_{0}$, but unbind everywhere on the membrane. Parameter $D_{3}$ describes the diffusion coefficient of the ligand.

calculated from the diffusive flux to a small sphere of radius $s$, which represents the receptor.

\section{RECEPTOR DIFFUSION MODEL}

In this model, depicted in Fig. 3, receptors are free to diffuse on the 2-dimensional (2D) membrane surface with diffusion coefficient $D_{2}$. A receptor located in the limited area $\Delta A$ centered at $\vec{x}_{0}$, can bind and unbind ligand of average concentration $\bar{c}$ with rates $k_{+} \bar{c}$ and $k_{-}$, respectively. When a receptor leaves this area and diffuses to the remaining large region of negligible ligand concentration, it can either release ligand or diffuse back in the area.

We consider a situation in which receptor diffusion has reached a stationary condition resulting in a uniform density $\rho_{0}$ of receptors on the membrane, and this condition is not changed by interaction with ligand molecules. Ligand binding and unbinding only affects the number of occupied and unoccupied receptors with respective densities $\rho_{b}$ and $\rho_{u}$, given by

$$
\rho_{0}=\rho_{b}(\vec{r})+\rho_{u}(\vec{r}),
$$

where $\vec{r}=(x, y)$ is a position on the $2 \mathrm{D}$ membrane surface. For purposes of comparison with the single immobile receptor model, we also assume that there is always exactly one receptor in the area $\Delta A$ around $\vec{x}_{0}=\left(\vec{r}_{0}, z_{0}\right)$, i.e. $\rho_{0}=1 / \Delta$.

The dynamics of such a system is captured by the fol- 
lowing set of coupled differential equations

$$
\begin{aligned}
& \frac{\partial \rho_{b}(\vec{r}, t)}{\partial t}=D_{2} \nabla^{2} \rho_{b}(\vec{r}, t)-k_{-} \rho_{b}(\vec{r}, t)+ \\
& k_{+}\left[\rho_{0}-\rho_{b}(\vec{r}, t)\right] c\left(\vec{r}, z_{0}, t\right) \Delta A \delta\left(\vec{r}-\vec{r}_{0}\right) \\
& \frac{\partial c(\vec{x}, t)}{\partial t}=D_{3} \nabla^{2} c(\vec{x}, t)-\delta\left(z-z_{0}\right)\left[\frac{\partial \rho_{b}(\vec{r}, t)}{\partial t}\right. \\
&\left.-D_{2} \nabla^{2} \rho_{b}(\vec{r}, t)\right]+k_{\infty} \delta\left(\vec{x}-\vec{x}_{\infty}\right)
\end{aligned}
$$

where rate constant $k_{\infty}$ ensures the replenishment of ligand molecules, $\vec{x}=(x, y, z)=(\vec{r}, z)$ indicates a location in 3D space. Specifically, $\rho_{b}(\vec{r}, t)$ is the density of bound receptors and $c(\vec{x}, t)$ is the ligand concentration, both depending on space and time. An additional equation for $\rho_{u}(\vec{r}, t)$ is not necessary due to receptor conservation in Eq. (8).

After normalization by the average density, using $n(\vec{r}, t)=\rho_{b}(\vec{r}, t) / \rho_{0}$ in Eq. (9), we obtain

$$
\begin{aligned}
& \frac{\partial n(\vec{r}, t)}{\partial t}=D_{2} \nabla^{2} n(\vec{r}, t)+k_{+}[1-n(\vec{r}, t)] c\left(\vec{r}, z=z_{0}, t\right) \Delta A \delta\left(\vec{r}-\overrightarrow{r_{0}}\right)-k_{-} n(\vec{r}, t) \\
& \frac{\partial c(\vec{x}, t)}{\partial t}=D_{3} \nabla^{2} c(\vec{x}, t)-\rho_{0} \delta\left(z-z_{0}\right)\left[\frac{\partial n(\vec{r}, t)}{\partial t}-D_{2} \nabla^{2} n(\vec{r}, t)\right]+k_{\infty} \delta\left(\vec{x}-\vec{x}_{\infty}\right) .
\end{aligned}
$$

These equations describe the coupled dynamics of the diffusing receptors and their occupancy with ligand. In summary, receptors can bind ligand molecules only within area $\Delta A$ but can release them anywhere on the membrane. The solution is provided in the following sections.

\section{NON-EQUILIBRIUM STATIONARY SOLUTION FOR RECEPTOR OCCUPANCY}

It is possible to extract an exact analytical expression for the stationary solution $\bar{n}(\vec{r})$ for the receptor occupancy from Eq. (10a). Setting the right-hand side to zero and Fourier Transforming both sides leads to

$$
\hat{\bar{n}}(\vec{q})=\frac{k_{+}\left[1-\bar{n}\left(\vec{r}_{0}\right)\right] \bar{c}\left(\vec{x}_{0}\right) \Delta A}{D_{2} q^{2}+k_{-}},
$$

where $\vec{q}$ is the wave vector of magnitude $q=|\vec{q}|$. The inverse Fourier Transform, upon introducing a cut-off $\Lambda \sim 1 / s$ due to the receptor size, leads to the following solution

$$
\bar{n}(\vec{r})=k_{+}\left[1-\bar{n}\left(\vec{r}_{0}\right)\right] \bar{c}\left(\vec{x}_{0}\right) \frac{\Delta A}{4 \pi D_{2}} \log \left(1+\frac{D_{2} \Lambda^{2}}{k_{-}}\right) .
$$

This stationary solution corresponds to a nonequilibrium condition since ligand is continuously taken away from area $\Delta A$. Setting $\Delta A=4 \pi / \Lambda^{2} \sim s^{2}$ and evaluating Eq. (12) in $\vec{r}_{0}$ leads therefore to the following value for the occupancy at $\vec{r}_{0}$

$$
\bar{n}\left(\vec{r}_{0}\right)=\frac{1}{1+\left[k_{+} \bar{c}\left(\vec{x}_{0}\right) \frac{\Delta A}{4 \pi D_{2}} \log \left(1+\frac{4 \pi D_{2}}{\Delta A k_{-}}\right)\right]^{-1}},
$$

which is plotted in Fig. 4 and, in the limit $D_{2} \rightarrow 0$, leads to the recovery of the immobile receptor solution Eq. (2),

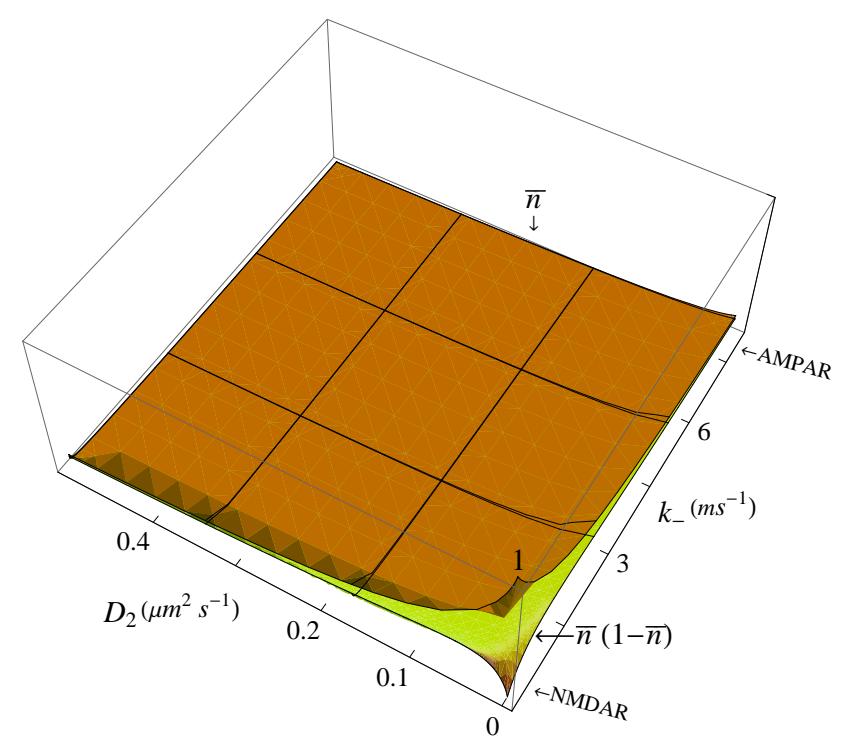

FIG. 4: (Color online) Receptor occupancy at $\vec{r}_{0}$. Eq. (13) for $\bar{n}$ is plotted as a function of lateral diffusion coefficient $D_{2}$ and unbinding rate $k_{-}$for a range of experimentally relevant values in neurotransmission (top surface). Specifically, the $k_{-}$values for AMPA and NMDA receptors are indicated (see Table I). Corresponding plot for $\bar{n}(1-\bar{n})$ is also shown (bottom surface).

namely $\bar{n}_{0}=k_{+} \bar{c} /\left(k_{-}+k_{+} \bar{c}\right)$. In order to quantify the deviation from equilibrium we apply Fick's first law to calculate the flux $\vec{J}$ of occupied receptors

$$
\vec{J}=D_{2} \vec{\nabla} \bar{n}(\vec{r}, t)
$$

out of region $\Delta A$ [19]. Integrating Eq. (14) over the border of $\Delta A$ allows us to define an effective "internalization 
rate" for removal of ligand in analogy to [12], via

$$
\begin{aligned}
k_{i}^{e} \bar{n} & =-\frac{D_{2}}{\Delta A} \oint \nabla \bar{n}(\vec{r}) d \vec{r}=-\frac{D_{2}}{\Delta A} \int_{\Delta A} \nabla^{2} \bar{n}(\vec{r}) d^{2} \vec{r} \\
& =\frac{1}{\Delta A} \int_{\Delta A} d^{2} \vec{r}\left[k_{+} \bar{c}(1-\bar{n}) \Delta A \delta(\vec{r})-k_{-} \bar{n}\right],
\end{aligned}
$$

where the second equality follows from integration by parts and the last equality results from the stationary condition for Eq. (10a). Carrying out the last integration leads to the following final expression for the effective internalization rate

$$
k_{i}^{e}=k_{+} \bar{c}\left(\frac{1-\bar{n}}{\bar{n}}\right)-k_{-}=\frac{4 \pi D_{2}}{\Delta A \log \left(1+\frac{4 \pi D_{2}}{\Delta A k_{-}}\right)}-k_{-},
$$

where we used Eq. (13) to express $\bar{n}$ in terms of the receptor diffusion coefficient. Using condition $\Delta A=4 \pi / \Lambda^{2}$ we can define a modified unbinding rate

$$
\kappa_{-}=k_{-}+k_{i}^{e}=\frac{k_{+} \bar{c}(1-\bar{n})}{\bar{n}}=\frac{D_{2} \Lambda^{2}}{\log \left(1+\frac{D_{2} \Lambda^{2}}{k_{-}}\right)}
$$

and extend the equilibrium condition Eq. (3) to the stationary non-equilibrium condition via an effective tem- perature $T_{e}$ defined by 12$]$

$$
\frac{k_{+} \bar{c}}{\kappa_{-}}=e^{\frac{F}{T_{e}}}
$$

This effective temperature allows us to generalize the ordinary FDT to a stationary non-equilibrium condition and to derive the receptor accuracy of sensing in the next section.

\section{ACCURACY OF SENSING}

Here we we derive the accuracy of sensing for diffusing receptors. Starting from Eq. (10) we consider an expansion to first order around the stationary solutions

$$
\begin{aligned}
n(\vec{r}, t) & =\bar{n}+\delta n(\vec{r}, t) \\
c(\vec{x}, t) & =\bar{c}+\delta c(\vec{x}, t),
\end{aligned}
$$

where $\bar{n}$ and $\bar{c}$ are the stationary solutions of Eq. (10), which, in this case, have a spatial dependence, i.e. $\bar{n}=$ $\bar{n}(\vec{r})$ and $\bar{c}=\bar{c}(\vec{x})$. Hence, the linearized equation for the receptor occupancy and ligand concentration are given by

$$
\begin{aligned}
& \frac{\partial[\delta n(\vec{r}, t)]}{\partial t}=D_{2} \nabla^{2} \delta n(\vec{r}, t)+\left[(1-\bar{n}) \delta c\left(\vec{r}, z_{0}, t\right)-\bar{c} \delta n(\vec{r}, t)+(1-\bar{n}) \bar{c} \frac{\delta k_{+}(t)}{k_{+}}-\bar{n} \frac{\delta k_{-}(t)}{k_{+}}\right] k_{+} \Delta A \delta\left(\vec{r}-\vec{r}_{0}\right)-k_{-} \delta n(\vec{r}, t) \\
& \frac{\partial[\delta c(\vec{x}, t)]}{\partial t}=D_{3} \nabla^{2} \delta c(\vec{x}, t)-\delta\left(z-z_{0}\right) \rho_{0}\left\{\frac{\partial[\delta n(\vec{r}, t)]}{\partial t}-D_{2} \nabla^{2} \delta n(\vec{r}, t)\right\}
\end{aligned}
$$

where we assumed that fluctuations in the binding/unbinding rate constants occur as well. This mathematical trick allows us to introduce fluctuations in the receptor-binding free energy and so to apply the FDT [9, 17]. Using the stationary non-equilibrium condition Eq. (18), we obtain the fluctuations in the rates and binding free energy

$$
\frac{\delta k_{+}}{k_{+}}-\frac{\delta k_{-}}{\kappa_{-}}=\frac{\delta F}{T_{e}} .
$$

Using this equation allows us to replace fluctuations in the rate constants in Eq. (20a) with fluctuations in the binding free energy, resulting in

$$
\begin{aligned}
& \frac{\partial[\delta n(\vec{r}, t)]}{\partial t}=D_{2} \nabla^{2} \delta n(\vec{r}, t)-k_{-} \delta n(\vec{r}, t)+\left[k_{+} \bar{c}(1-\bar{n}) \frac{\delta F}{T_{e}}\right. \\
& \left.+k_{+}(1-\bar{n}) \delta c\left(\vec{r}, z_{0}, t\right)-k_{+} \bar{c} \delta n(\vec{r}, t)\right] \Delta A \delta\left(\vec{r}-\overrightarrow{r_{0}}\right) .
\end{aligned}
$$

Fourier Transforming Eq. (22) and setting $q=|\vec{q}|$, we solve for $\delta \hat{n}(\vec{q}, \omega)$ and obtain

$$
\delta \hat{n}(\vec{q}, \omega)=G\left(\omega, \vec{r}_{0}, z_{0}\right) \frac{e^{i \vec{q} \cdot \overrightarrow{r_{0}}}}{D_{2} q^{2}+k_{-}-i \omega},
$$


where, for convenience of calculation, we have defined the following function

$$
\begin{gathered}
G\left(\omega, \vec{r}_{0}, z_{0}\right)=\left[k_{+}(1-\bar{n}) \delta \hat{c}\left(\vec{r}_{0}, z_{0}, \omega\right)-k_{+} \bar{c} \delta \hat{n}\left(\vec{r}_{0}, \omega\right)+\right. \\
\left.(1-\bar{n}) \bar{c} \frac{\delta F(\omega)}{T_{e}}\right] \Delta A .
\end{gathered}
$$

Inverse Fourier Transforming Eq. (23) leads to the following expression for the spectrum of the fluctuations in occupancy

$$
\begin{aligned}
\delta \hat{n}\left(\vec{r}_{0}, \omega\right) & =\int \frac{d \vec{q}}{(2 \pi)^{2}} e^{-i \vec{q} \cdot \vec{r}_{0}} \delta \hat{n}(\vec{q}, \omega) \\
& =G\left(\omega, \vec{r}_{0}, z_{0}\right) \int \frac{d \vec{q}}{(2 \pi)^{2}} \frac{1}{D_{2} q^{2}+k_{-}-i \omega} \\
& =G\left(\omega, \vec{r}_{0}, z_{0}\right) \Sigma_{1}(\omega),
\end{aligned}
$$

where we defined

$$
\Sigma_{1}(\omega)=\int \frac{d \vec{q}}{(2 \pi)^{2}} \frac{1}{D_{2} q^{2}+k_{-}-i \omega}
$$

with the integral provided in Appendix A. In order to remove the ligand concentration in Eq. (24), we Fourier Transform Eq. (20b), leading to

$$
\delta \hat{c}(\vec{q}, \omega)=\rho_{0} \frac{i \omega-D_{2} q^{2}}{D_{3}\left(q^{2}+q_{\perp}^{2}\right)-i \omega} \delta \hat{n}(\vec{q}, \omega) e^{i q_{\perp} z_{0}} .
$$

Inserting Eq. (23) in Eq. (27) and inverse Fourier Transforming leads to the following expression for the spectrum of the fluctuations in ligand concentration

$$
\begin{aligned}
\delta \hat{c}\left(\vec{r}_{0}, z_{0}, \omega\right) & =\int \frac{d q_{\perp}}{2 \pi} e^{-i q_{\perp} z_{0}} \int \frac{d \vec{q}}{(2 \pi)^{2}} e^{-i \vec{q} \cdot \vec{r}_{0}} \delta \hat{c}(\vec{q}, \omega) \\
& =G\left(\omega, \vec{r}_{0}, z_{0}\right) \rho_{0} \Sigma_{2}(\omega)
\end{aligned}
$$

with

$\Sigma_{2}(\omega)=\int \frac{d q_{\perp}}{2 \pi} \int \frac{d \vec{q}}{(2 \pi)^{2}} \frac{i \omega / D_{2}-q^{2}}{D_{3}\left(q^{2}+q_{\perp}^{2}\right)-i \omega} \frac{e^{-i \vec{q} \cdot \vec{r}_{0}}}{q^{2}+\frac{k_{-}}{D_{2}}-\frac{i \omega}{D_{2}}}$

provided in Appendix B. Inserting Eqs. (25) and (28) in Eq. (24) leads to a closed equation for the function $G\left(\omega, \vec{r}_{0}, z_{0}\right)$

$$
\begin{aligned}
& G\left(\omega, \vec{r}_{0}, z_{0}\right)=\left[k_{+}(1-\bar{n}) \rho_{0} \Sigma_{2}(\omega) G\left(\omega, \vec{r}_{0}, z_{0}\right)+\right. \\
& \left.-k_{-} \bar{c} \Sigma_{1}(\omega) G\left(\omega, \vec{r}_{0}, z_{0}\right)+k_{+} \bar{c}(1-\bar{n}) \frac{\delta \hat{F}(\omega)}{T_{e}}\right] \Delta A,
\end{aligned}
$$

which can be solved for $G\left(\omega, \vec{r}_{0}, z_{0}\right)$. This leads to

$$
G\left(\omega, \vec{r}_{0}, z_{0}\right)=\frac{k_{+} \bar{c}(1-\bar{n}) \Delta A \delta \hat{F}(\omega) / T_{e}}{1-k_{+}(1-\bar{n}) \Sigma_{2}(\omega)+k_{+} \bar{c} \Sigma_{1}(\omega) \Delta A},
$$

a

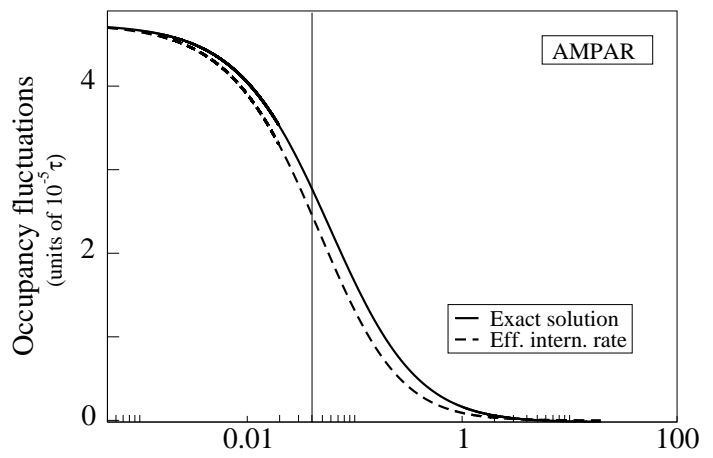

b

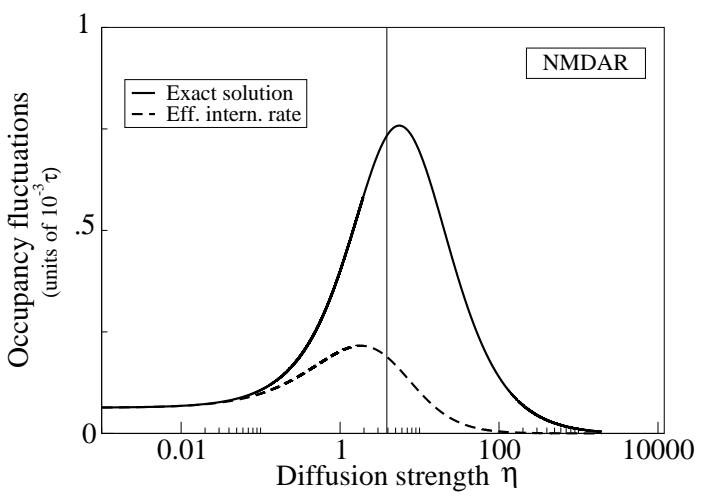

FIG. 5: Receptor-occupancy fluctuations $\left\langle(\delta n)^{2}\right\rangle_{\tau}$ in units of $\tau$ as a function of $\eta=16 D_{2} /\left(s^{2} k_{-}\right)$for AMPAR (a) and NMDAR (b). Shown are (solid line) plot of $\left\langle(\delta n)^{2}\right\rangle_{\tau}$ as given by Eqs. (34) and (36), (dashed line) plot of Eq. (37) using the effective internalization rate Eq. (16), and (vertical thin line) experimental value of $\eta$ for each receptor type (see Table I for all parameters value). Glutamate concentration was set to $\bar{c}=0.1 \mathrm{mM}$, here and in Fig. 6 . In order to produce a significant effect from ligand rebinding, we used $D_{3}=0.1 \mu \mathrm{m}^{2} / \mathrm{s}$ here and in subsequent plots, as may result from binding of glutamate to neuroligins, neurexins and other cleft proteins.

where condition $\rho_{0}=1 / \Delta A$ was applied. Using Eqs. (25) and (31), a final expression for the response is obtained

$$
\begin{aligned}
\frac{\delta \hat{n}\left(\vec{r}_{0}, \omega\right)}{\delta \hat{F}(\omega)} & =\frac{G\left(\omega, \vec{r}_{0}, z_{0}\right) \Sigma_{1}(\omega)}{\delta \hat{F}(\omega)} \\
& =\frac{1}{T_{e}} \frac{k_{+} \bar{c}(1-\bar{n}) \Delta A \Sigma_{1}(\omega)}{1-k_{+}(1-\bar{n}) \Sigma_{2}(\omega)+k_{+} \bar{c} \Sigma_{1}(\omega) \Delta A}
\end{aligned}
$$

Applying the generalized FDT we derive the spectrum of the fluctuations in receptor occupancy $\delta \hat{n}\left(\vec{r}_{0}, \omega\right)$ from the response, namely

$$
S(\omega)=\frac{2 k T_{e}}{\omega} \operatorname{Im}\left[\frac{\delta \hat{n}\left(\vec{r}_{0}, \omega\right)}{\delta \hat{F}(\omega)}\right] .
$$

By replacing the expressions for $\Sigma_{1}(\omega)$ and $\Sigma_{2}(\omega)$ in Eq. (32), taking the imaginary part and setting $\omega \simeq 0$, we 
obtain for the zero-frequency limit of the power spectrum

$$
\begin{aligned}
& S_{\eta}(0)=\frac{k_{+} \bar{c}(1-\bar{n}) \Delta A \Lambda^{2}}{2 \pi k_{-}^{2}(1+\eta)\left[1+\frac{k_{+} \bar{c}}{k_{-}} L_{\eta}+\frac{k_{+}(1-\bar{n}) \Lambda}{8 \pi D_{3}}\left(1-A_{\eta}\right)\right]^{2}} \times \\
& {\left[1+\frac{k_{+}(1-\bar{n}) \Lambda}{16 \pi D_{3}}\left(1+\frac{D_{3} \Lambda^{2}-\eta k_{-}}{D_{3} \Lambda^{2} /(1+\eta)} A_{\eta}+\frac{1-A_{\eta}}{L_{\eta} / 2}\right) L_{\eta}\right],}
\end{aligned}
$$

where we conveniently defined the following functions of the dimensionless parameter $\eta=D_{2} \Lambda^{2} / k_{-}$

$$
L_{\eta} \equiv \frac{\log (1+\eta)}{\eta}, \quad A_{\eta} \equiv \frac{\arctan \sqrt{\eta}}{\sqrt{\eta}} .
$$

Note that in Eq. (34), occupancy $\bar{n}$ also depends on $\eta$ via Eq. (13). The zero-frequency spectrum is related to the receptor occupancy fluctuations, averaged over a time $\tau \gg k_{-}^{-1},\left(k_{+} \bar{c}\right)^{-1}$, by the following relation:

$$
\left\langle(\delta n)^{2}\right\rangle_{\tau} \simeq S_{\eta}(0) / \tau
$$

In Fig. 5 we plot the occupancy fluctuations, given by Eq. (36) as a function of $\eta$, i.e. the effective diffusion strength. This is done for the two main receptors involved in neurotransmission, namely AMPAR and NMDAR, with parameters taken from Table I. For AMPAR, the effect of diffusion is that of decreasing the fluctuations in occupancy due to reduced overcounting of previously bound molecules (Fig. 5a). For small unbinding rates $k_{-}$though, such as occurs for the NMDA receptor, an actual increase in the noise is observed for a range of physiologically relevant diffusion strengths.

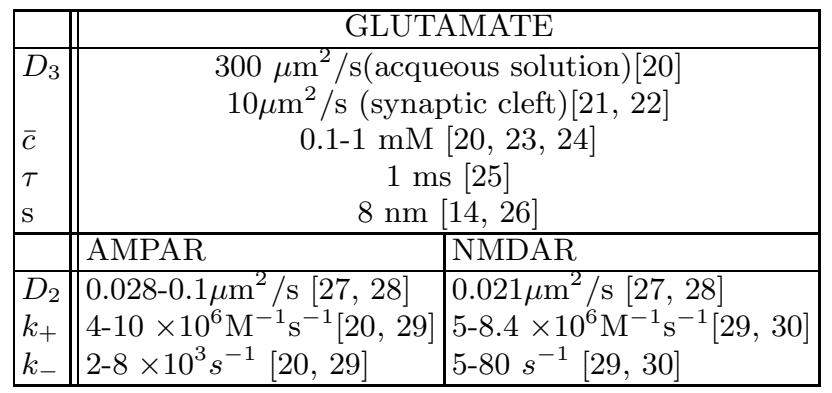

TABLE I: Summary of experimentally determined parameters for glutamate ligand (top), AMPA receptor (bottom left) and NMDA receptor (bottom right).
In order to make the origin of this effect more transparent, we also plot the occupancy fluctuations in Fig. 5 as calculated for the case of receptor internalization [12]

$$
\left\langle(\delta n)^{2}\right\rangle_{\tau} \simeq \frac{2 \bar{n}^{2}(1-\bar{n})}{k_{+} \bar{c} \tau}
$$

where $\bar{n}=k_{+} \bar{c} /\left(k_{+} \bar{c}+k_{-}+k_{i}^{e}\right)$, with $k_{i}^{e}$ defined in Eq. (16). The experimental unbinding rate of NMDAR leads to values for $\bar{n}$ such that starting from $D_{2}=0$, the function $\bar{n}(1-\bar{n})$ increases, reaches a maximum and then decreases again. Such a maximum is avoided by AMPAR whose spectrum monotonically decreases when $D_{2}$ increases (see Fig. 4). The comparison between receptor diffusion and receptor internalization in Fig. 5 shows qualitatively similar results, confirming the effective ligand removal by receptor diffusion.

\section{A. Equilibrium limit for vanishing $D_{2}$}

Here we show that Eq. (34) leads to the expression for the single receptor obtained in Refs. [9, 18] in the limit for $D_{2} \rightarrow 0$, i.e. $\eta \rightarrow 0$. Taking the limit and using $\Delta A=4 \pi / \Lambda^{2}$, we indeed obtain

$$
\lim _{\eta \rightarrow 0} S_{\eta}(0)=\frac{2 k_{+} \bar{c}\left(1-\bar{n}_{0}\right)}{\left(k_{-}+k_{+} \bar{c}\right)^{2}}\left[1+\frac{k_{+}\left(1-\bar{n}_{0}\right) \Lambda}{8 \pi D_{3}}\right],
$$

which is identical to the result for the single receptor for $\Lambda=4 / s$.

\section{B. Near-equilibrium result for small $D_{2}$}

We derive here the exact result, Eq. (34), in the limit of slow receptor diffusion on the membrane, using $\eta=$ $D_{2} /\left(s^{2} k_{-}\right)$as a small parameter. Specifically, we are interested in the accuracy of sensing, which is derived from the normalized variance for the ligand concentration of ligand as in [9]

$$
\frac{\left\langle(\delta c)^{2}\right\rangle_{\tau}}{\bar{c}^{2}}=\frac{\left\langle(\delta n)^{2}\right\rangle_{\tau}}{\bar{n}^{2}(1-\bar{n})^{2}} \simeq \frac{1}{\bar{n}^{2}(1-\bar{n})^{2}} \frac{S_{\eta}(0)}{\tau},
$$

where the right-hand term follows from Eq. (36). Expansion to first order in parameter $\eta$ leads to

$$
\frac{\left\langle(\delta c)^{2}\right\rangle_{\tau}}{\bar{c}^{2}}=\frac{2}{k_{+} \bar{c}\left(1-\bar{n}_{0}\right) \tau}\left(1-8 \bar{n}_{0} \eta\right)+\frac{1}{\pi s D_{3} \bar{c} \tau}\left[1-\left(\frac{16 k_{-}^{2} \bar{n}_{0}^{2}}{3 \pi k_{+} \bar{c}^{2} D_{3} s}+8 \bar{n}_{0} \frac{3 k_{-}-k_{+} \bar{c}}{3 k_{+} \bar{c}}+\frac{k_{-}}{2 D_{3}} s^{2}\right) \eta\right] .
$$

Comparison with Eq. (6a) shows that the correction due to lateral receptor diffusion is twofold to first or- der: (i) the first term is reduced due to a reduction in $\bar{n}$, 
a

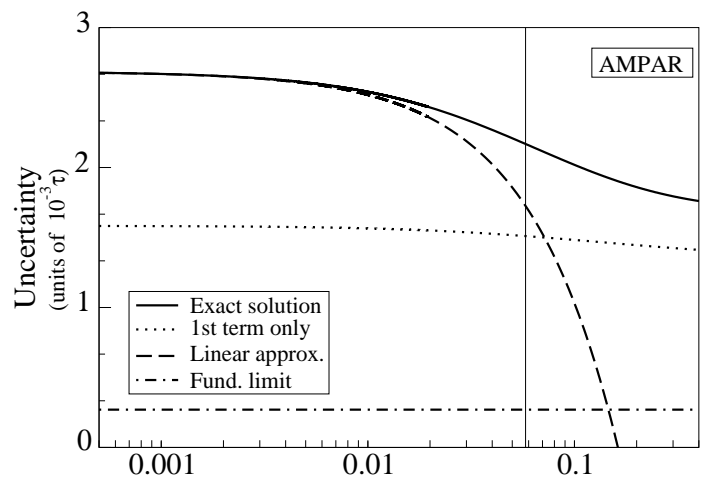

b

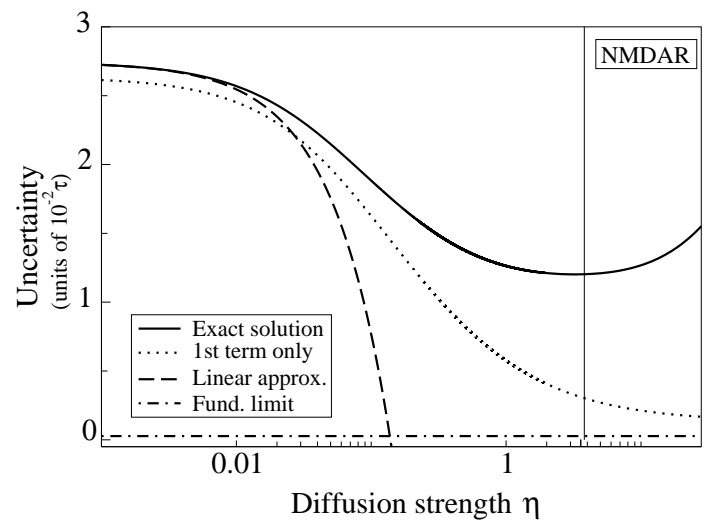

a

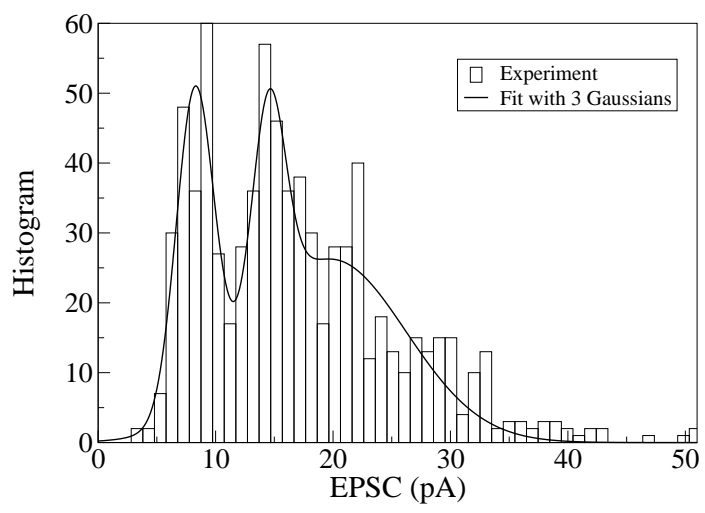

b

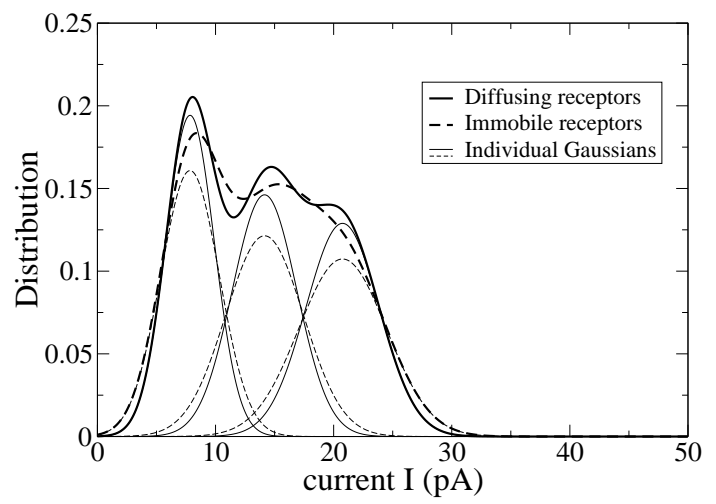

FIG. 6: Uncertainty $\left\langle(\delta c)^{2}\right\rangle_{\tau} / \bar{c}^{2}$ as a function of $\eta=$ $16 D_{2} /\left(s^{2} k_{-}\right)$for the AMPA receptor (a) and the NMDA receptor (b) in units of $\tau$. Shown are (solid line) plot of Eq. (39) as obtained from the exact solution Eq. (34), (dashed line) linear approximation for small $\eta$, and (dotted line) contribution to the accuracy of sensing from only the first term in Eq. (40). Also shown are (vertical thin line) the experimental value of $\eta$ for each receptor (see Table I) and (horizontal dot-dashed line) the fundamental physical limit Eq. (7)

(ii) the second term is reduced for most parameter values due to a reduction in rebinding of already measured ligand molecules.

In Fig. 6 we plot the uncertainty in sensing for both AMPAR and NMDAR. In both cases the total effect of lateral diffusion is that of decreasing the uncertainty in the concentration and therefore of increasing the accuracy of sensing. Receptor diffusion for AMPAR (Fig. 6a) mainly reduces the rebinding term, while for NMDAR (Fig. 6b) the first term from binding and unbinding is reduced. In the latter case, the maximum in the occupancy fluctuations (see Fig. 5) is removed through $\bar{n}^{2}(1-\bar{n})^{2}$ in the denominator of Eq. (39). For large values of $\eta$, the occupancy fluctuations and hence the accuracy of sensing become unphysical (zero, i.e. below the physical limit, or diverge) as the effective temperature breaks down far from equilibrium. (For large $\eta$, receptors remove ligand molecules efficiently, which introduces large non-equilibrium ligand fluxes. These cannot be rep-
FIG. 7: Quantal transmission. (a) Histogram of experimental EPSC (excitatory post-synaptic current) data taken from Ref. [34] and fitted by three Gaussian distributions. (b) Distribution of predicted EPSCs. Shown are individual Gaussian distributions corresponding to one, two and three vesicles (from left to right; thin lines), as well as envelope functions (sums with equal weighting; thick lines). Calculation is done for AMPA receptors using Eqs. (41, 42), Eq. (39) for the variances of diffusing (thin solid lines) and Eq. (6a) for immobile (thin dashed lines) receptors, assuming glutamate concentration $\bar{c}_{n}=c_{b}+n c_{v}$ for $n=1,2,3$ vesicles, background $c_{b}=0.02 \mathrm{mM}$, vesicle concentration $c_{v}=0.04 \mathrm{mM}$ and $N=100$ receptors 44, 45]. Furthermore, Eq. (41) with parameters from Refs. [34, 35] was used.

resented by an effective temperature.)

In summary, lateral receptor diffusion affects the accuracy of sensing in two ways: (i) by reducing the stationary value for the receptor occupancy, and (ii) by reducing the noise from rebinding of already measured ligand molecules.

\section{QUANTAL TRANSMISSION}

A remarkable property of synaptic transmission is that the amplitude of the synaptic response, i. e. the excitatory post-synaptic current (EPSC), varies by an integral 
multiple of a quantum. This property, first unveiled by the pioneering work of Katz [31], was traced to the formation of synaptic vesicles and subsequent pore formation. The latter leads to a release of glutamate into the synaptic cleft [32]. This quantization is considered a way to minimize the variability of the response from each contributing synapse and therefore to allow efficient neural computation. This variability is quantified by the coefficient of variation $(\mathrm{CV})$ defined as the standard deviation devided by the mean value. The reason for a large $\mathrm{CV}$ is controversial (cf. Fig. 7a), but can be attributed to a variation in the size of the vesicles carrying glutamate, or to the fact that large EPSCs are generated by multiple vesicles [13, 46]. The experimental detection of EPSCs with an amplitude distribution of more than one peak is consistent with the latter explanation [33].

In Fig. 7a, the experimental data taken from Ref. 34] show EPSC peaks as a result of repetitive suprathreshold stimulation of granule cell somata in CA3 pyramidal cells. Some stimuli did not evoke an EPSC (termed failures). The histogram of successful events can be fitted with three Gaussian distributions, showing the quantal nature of the EPSC. The peaks of the Gaussians correspond to the discrete multiple values 7,14 , and $21 \mathrm{pA}$, respectively.

We can compare this distribution to the result predicted by the accuracy of sensing. Specifically, we would like to know if fast diffusion helps resolve the EPSC peaks. A simple model based on the structural properties of AMPAR was previously used to derive the dynamics of glutamate binding and unbinding, as well as channel opening and closing [34]. Based on this model the following relation connecting the current through the ion channel of AMPAR and the concentration of glutamate was deduced:

$$
I=I_{0} \frac{1}{1+(\lambda / c)^{n}},
$$

where $I_{0}, \lambda$, and the Hill coefficient $n$ are parameters provided in Ref. [34, 35]. Based on Eq. (41) the following relation connecting the width in the distribution of EPSC currents to the fluctuations in glutamate concentration can be derived:

$$
\delta I=I_{0} n \lambda \frac{(\lambda / c)^{n-1}}{\left[1+(\lambda / c)^{n}\right]^{2}} \delta c .
$$

We further assume that a number $N$ of receptors independently contribute to the measurement, i. e.

$$
\frac{\left\langle(\delta c)^{2}\right\rangle_{\tau, N}}{\bar{c}^{2}}=\frac{\left\langle(\delta c)^{2}\right\rangle_{\tau}}{N \bar{c}^{2}}
$$

with the uncertainty of a single receptor given by Eq. (39). Averaging over $N$, therefore, further reduces the uncertainty in Eq. (43) and hence increases the accuracy of sensing.

Figure $7 \mathrm{~b}$ shows that the distributions of predicted EP$\mathrm{SCs}$ is in qualitative agreement with the experiments.
From our calculations we found that the effect of lateral receptor diffusion is to increase the resolution of peaks and so also the ability of the synapse to count the number of vesicles released into the synaptic cleft. Note that in order to resolve the EPSC peaks experimentally, the probability of vesicle release from the presynaptic side was drastically reduced. This was achieved by modulating the ratio of $\mathrm{Ca}^{+} / \mathrm{Mg}^{+}$ions in the extracellular solution, resulting in a 50-60\% reduction of peak currents 34.

\section{DISCUSSION AND CONCLUSIONS}

Our previous work on the fundamenal physical limit of ligand sensing [12] led us to conclude that receptor diffusion, by removing bound ligand from a region of interest, may reduce the local overcounting of ligand molecules, therefore potentially increasing the local accuracy of sensing. In this paper we set out to investigate this possibility by constructing a mathematical model, that contains all the necessary ingredients to describe the role of receptor diffusion in ligand sensing. We have considered a receptor, that can bind and unbind ligand molecules as well as diffuse on a 2D membrane. Ligand is allowed to diffuse in 3D space. Using this model we derived the fluctuations in receptor occupancy in the area of interest via a generalization of the FDT with the introduction of an effective temperature [12]. This ultimately allowed us to derive an equation for the accuracy of sensing.

We applied our model to the biologically relevant case of glutamate receptors, which are responsible for transmitting action potentials at neural synapses. We found that for AMPAR and NMDAR, our model shows that the lateral diffusion of receptors increases their accuracy of sensing and hence may allow synapses to count the number of released vesicles. However, the local occupancy fluctuations are decreased only for AMPAR; for NMDAR these fluctuations are increased. This difference is due to the smaller unbinding rate of the latter receptor. Consequently, the accuracy of sensing for NMDAR is an order of magnitude smaller than for AMPAR. It is important to remark that these results are consistent with the different roles these receptors play. In fact, the transmission of an excitatory potential through a synapse occurs in several steps. Initially, it is AMPAR which senses glutamate and, by letting in ions, starts a small depolarization of the membrane. This in turn allows NMDAR to open its channel and start an even bigger influx of ions (especially $\mathrm{Ca}^{+}$). This stimulates the production of more AMPARs and so increases the strength and sensitivity of the synapses. Sensitivity to glutamate is important for AMPAR, while the roles of NMDAR are coincidence detection and amplification, important for long-term potentiation and memory.

In order to derive the accuracy of sensing for diffusing receptors, we made a number of symplifying assumptions. 
We neglected fluctuations in the total receptor density and vesicle size, assumed to be constant [13, 46]. Furthermore, we assumed a constant ligand concentration in the hotspot area $\Delta A$ during the receptor measurement time, while in reality the concentration profile will broaden out. Additionally, we made the simplifying assumption that multiple vesicles are released in the same spot, so that released glutamate concentrations add up, and that other effects such as spillover from neighboring cells can be neglected [42, 43]. Finally, we introduced the effective temperature $T_{e}$ to generalize the FDT to non-equilibrum processes [12, 36 39]. The approximate nature of $T_{e}$, for which we neglected any potential time or frequency dependence, limits the quantitative validity of our model to small deviations from equilibrium. In fact, for large receptor diffusion coefficients, the occupancy fluctuations and hence the accuracy of sensing become unphysical. Nevertheless, the mapping of receptor diffusion to an effective internalization process, already studied in Ref. [12], provides confidence in our method.

In conclusion, in this paper we highlighted the role of diffusion in increasing the accuracy of sensing. This might be important for synaptic counting of glutamate vesicles. This hypothesis can be tested experimentally by reducing the mobility of receptors. This can be achieved by cross-linking or addition of cholesterol. Alternatively, the glutamate diffusion costant can be reduced by adding dextran. Such perturbations should lead to a broadening of the EPSC peaks, although signaling may be affected as well. Similarly to AMPAR [15], an increase in receptor diffusion upon ligand binding was recently also observed in the pseudopods of migrating Dictyostelium discoideum cells [40]. This phenomenon was attributed to signal amplification. However, since cells may sense locally in pseudopods, at least in shallow gradients [41], receptor diffusion may also be responsible for increasing the accuracy of sensing.

\section{Acknowledgments}

We would like to thank Aldo Faisal and Peter Jonas for helpful comments. We acknowledge financial support from Biotechnological and Biological Sciences Research Council grant BB/G000131/1 and the Centre for Integrated Systems Biology at Imperial College.

\section{Appendix A: Calculation of $\Sigma_{1}(\omega)$}

We devote this Appendix to the calculation of the integral $\Sigma_{1}(\omega)$ in Eq. (26). $\Sigma_{1}(\omega)$ is given by:

$\Sigma_{1}(\omega)=\int \frac{d \vec{q}}{(2 \pi)^{2}} \frac{1 / D_{2}}{q^{2}+\frac{k_{-}}{D_{2}}-\frac{i \omega}{D_{2}}}=\int_{0}^{\infty} \frac{d q}{2 \pi} \frac{q / D 2}{q^{2}+\frac{k_{-}}{D_{2}}-\frac{i \omega}{D_{2}}}$,

which is divergent. Hence, we introduce a cut-off $\Lambda \sim 1 / s$ to account for the finite dimension $s$ of the receptor. This procedure leads to the final result:

$$
\Sigma_{1}(\omega)=\int_{0}^{\Lambda} \frac{d q}{2 \pi} \frac{q / D_{2}}{q^{2}+\frac{k_{-}}{D_{2}}-\frac{i \omega}{D_{2}}}=\frac{\log \left(1+\frac{D_{2} \Lambda^{2}}{k_{-}-i \omega}\right)}{4 \pi D_{2}} .
$$

\section{Appendix B: Calculation of $\Sigma_{2}(\omega)$}

In this appendix, we calculate $\Sigma_{2}(\omega)$ in Eq. (29). Without loss of generality, we set $\left(x_{0}, y_{0}, z_{0}\right)=(0,0,0)$ and obtain:

$$
\begin{aligned}
& \Sigma_{2}(\omega)=\int_{0}^{\infty} \frac{d q_{\perp}}{2 \pi} \int_{0}^{\infty} \frac{q d q}{2 \pi} \frac{i \omega / D_{2}-q^{2}}{\left(q^{2}+q_{\perp}^{2}\right)-\frac{i \omega}{D_{3}}} \frac{1 / D_{3}}{q^{2}+\frac{k_{-}}{D_{2}}-\frac{i \omega}{D_{2}}} \\
& =\int_{0}^{\infty} \frac{d q_{\perp}}{2 \pi} \int_{0}^{\infty} \frac{d q}{2 \pi} \frac{q / D_{3}}{\left(q^{2}+q_{\perp}^{2}\right)-\frac{i \omega}{D_{3}}}\left(\frac{k_{-} / D_{2}}{q^{2}+\frac{k_{-}}{D_{2}}-\frac{i \omega}{D_{2}}}-1\right) \\
& =I_{1}(\omega)-I_{2}(\omega),
\end{aligned}
$$

where

$$
\begin{aligned}
& I_{1}(\omega)=\int_{0}^{\infty} \frac{d q_{\perp}}{2 \pi} \int_{0}^{\infty} \frac{q d q}{2 \pi} \frac{1 / D_{3}}{\left(q^{2}+q_{\perp}^{2}\right)-\frac{i \omega}{D_{3}}} \frac{k_{-} / D_{2}}{q^{2}+\frac{k_{-}}{D_{2}}-\frac{i \omega}{D_{2}}} \\
& =\int_{0}^{\Lambda} \frac{q d q}{8 \pi} \frac{i / D_{3}}{\sqrt{i \omega / D_{3}-q^{2}}} \cdot \frac{k_{-} / D_{2}}{q^{2}+\frac{k_{-}}{D_{2}}-i \frac{\omega}{D_{2}}} \\
& =\frac{k_{-} /\left(8 \pi D_{3}\right)}{\sqrt{\frac{k_{-}}{D_{2}}-i \omega\left(\frac{1}{D_{2}}-\frac{1}{D_{3}}\right)}}\left[\arctan \left(\sqrt{\frac{\Lambda^{2}-i \omega / D_{3}}{\frac{k_{-}}{D_{2}}-i \omega\left(\frac{1}{D_{2}}-\frac{1}{D_{3}}\right)}}\right)\right. \\
& \left.-\arctan \left(\sqrt{\frac{k_{-}-i \omega / D_{3}}{D_{2}}-i \omega\left(\frac{1}{D_{2}}-\frac{1}{D_{3}}\right)}\right)\right]
\end{aligned}
$$

and

$$
\begin{aligned}
& I_{2}(\omega)=\int_{0}^{\infty} \frac{d q_{\perp}}{2 \pi} \int_{0}^{\infty} \frac{q d q}{2 \pi} \frac{1}{-i \omega+D_{3}\left(q^{2}+q_{\perp}^{2}\right)} \\
& =\frac{1}{D_{3}} \int_{0}^{\Lambda} \frac{q d q}{8 \pi} \frac{i}{\sqrt{-q^{2}+i \omega / D_{3}}} \\
& =\frac{1}{8 \pi D_{3}}\left(\sqrt{-\frac{i \omega}{D_{3}}}-\sqrt{\Lambda^{2}-\frac{i \omega}{D_{3}}}\right)
\end{aligned}
$$

using a cut off to account for the finite size of the receptor. Taken together, the integral $\Sigma_{2}(\omega)$ is given by:

$$
\begin{aligned}
& \Sigma_{2}(\omega)=I_{1}(\omega)-I_{2}(\omega)=\left\{\left(\sqrt{\Lambda^{2}-\frac{i \omega}{D_{3}}}-\sqrt{-\frac{i \omega}{D_{3}}}\right)+\right. \\
& +\frac{k_{-} / D_{2}}{\sqrt{\frac{k_{-}}{D_{2}}-i \omega\left(\frac{1}{D_{2}}-\frac{1}{D_{3}}\right)}}\left[\arctan \left(\sqrt{\frac{\Lambda^{2}-i \omega}{\frac{k_{-}}{D_{2}}-i \omega\left(\frac{1}{D_{2}}-\frac{1}{D_{3}}\right)}}\right)\right. \\
& \left.\left.-\arctan \left(\sqrt{\frac{-i \omega}{\frac{k_{-}}{D_{2}}-i \omega\left(\frac{1}{D_{2}}-\frac{1}{D_{3}}\right)}}\right)\right]\right\} \frac{1}{8 \pi D_{3}} .
\end{aligned}
$$


[1] A. Raj and A. van Oudenaarden, Cell. 135, 216 (2008).

[2] R. C. Yu et al., Nature 456, 755 (2008).

[3] M. S. Samoilov1, G. Price, and A. P. Arkin, Sci. STKE 2006, re17 (2006).

[4] T. Gregor et al., Cell 130, 153 (2007).

[5] H. Mao, P.S. Cremer, M. D. Manson, Proc. Natl. Acad. Sci. USA 100, 5449 (2003).

[6] R. A. Arkowitz Trends Cell. Biol. 9, 20 (1999).

[7] J. E. Segall, Proc. Natl. Acad. Sci. USA 90, 8332 (1993).

[8] H. C. Berg and E. M. Purcell, Biophys. J., 20, 193 (1977)

[9] W. Bialek and S. Setayeshgar, Proc. Natl. Acad. Sci. USA 102, 10040 (2005).

[10] R. G. Endres and N. S. Wingreen, Proc. Natl. Acad. Sci. USA 105, 15749 (2008).

[11] R. G. Endres and N. S. Wingreen, Phys. Rev. Lett. 103, 158101 (2009).

[12] G. Aquino and R. G. Endres, Phys. Rev. E 81, 021909 (2010).

[13] A.A. Faisal, L.P.J. Selen and D.M. Wolvert, Nature 9, 292 (2008).

[14] B. Alberts, A. Johnson, J. Lewis, M. Raff, K. Roberts and Peter Walter, Molecular Biology of the Cell, Garland Publishing Inc. US, fifth edition (2007).

[15] M. Renner et al., Curr. Opin. Neurobio. 18, 532 (2008).

[16] M. Heine et al. , Science 320, 201 (2008).

[17] R. Kubo, Rep. Prog. Phys. 29, 255 (1966).

[18] R. G. Endres and N.S. Wingreen, Progr. Biophys. Mol. Biol. 100, 33 (2009).

[19] H. C. Berg, Random Walks in Biology, Princeton University Press (1993).

[20] M. Postlethwaite et al., J. Physiol. 579, 69 (2007).

[21] T. A. Nielsen, D. A. Di Gregorio and R. A. Silver, Neuron 42, 757 (2004); E. É. Saftenku, J. Theor. Biol. 234, 363 (2005).

[22] D. Choquet and A. Triller, Nat. Rev. Neurosci. 4, 251 (2003); D. Holcman and A. Triller, Biophys. J. 91, 2405
(2006).

[23] F. Ventriglia and V. Di Maio, Biosys. 58, 67 (2000).

[24] P. Jonas, News Physiol. Sci. 15, 83 (2000).

[25] Molecular Biology of the Cell M. J. T. Fitzgerald, G. Gruener and E. Mtui, Clinical Neuroanatomy and Neuroscience, Saunders Elsevier Ed., fifth edition (2007).

[26] T. Nakagawa, Y. Cheng, M. Sheng and T. Walz, Biol. Chem. 387, 179 (2006).

[27] L. Groc et al., Nature Neurosci. 7, 695 (2004); L. Groc et al., Proc. Nat. Acad. of Sci. USA 103, 18769 (2006).

[28] A. J. Borgdorff and D. Choquet, Nature 417, 649 (2002).

[29] D. Attwell and A. Gibb, Neuroscience 6, 841 (2005).

[30] K. Erreger and S. F. Traynelis, J. Physiol. 569, 381 (2005).

[31] B. Katz, The Release of Neural Neurotransmitter Substances, Liverpool University Press, Liverpool (1969).

[32] J. E. Heuser, J. Cell Biol. 81, 275 (1979).

[33] M. J. Wall and M. M. Usowicz, Nature Neurosci. 1, 675 (1998).

[34] P. Jonas and B.Sakmann, J. Physiol. 472, 615 (1993)

[35] P. Jonas and B.Sakmann, J. Physiol. 455, 143 (1992),

[36] L. F. Cugliandolo, J. Kurchan, and L. Peliti, Phys. Rev. E 55, 3898 (1997).

[37] Th. M. Nieuwenhuizen, Phys. Rev. Lett. 80, 5580 (1998).

[38] A. Crisanti and F. Ritort, J. Phys. A 36, R181 (2003).

[39] L. Leuzzi, J. of Non-Cryst. Solids 355, 686 (2009).

[40] S. de Keijzer et al., J. Cell. Sci. 121, 1750 (2008).

[41] N. Andrew and R. H. Insall, Nat. Cell. Biol. 9, 193 (2007).

[42] J.J. Lawrence et al., J. of Phys. 554, 175 (2003).

[43] P. B. Sargent et al, J. of Neurosci. 25, 8173 (2005).

[44] D. M. Kullmann, M-Y Min, F. Asztely, D.A. Rusakov, Phil. Trans. R. Soc. Lond. B 354, 395 (1999).

[45] J. R. Cottrell et al., J. Neurophysiol. 84, 1573 (2000).

[46] K. M. Franks, C.F. Stevens, T. K. Sejnowski, J. Neurosci. 23, 3186 (2003). 